\title{
Global Exponential Stabilization for a Class of Uncertain Nonlinear Control Systems Via Linear Static Control
}

\author{
Yeong-Jeu Sun \\ Professor, Department of Electrical Engineering, I-Shou University, Kaohsiung, Taiwan
}

\begin{abstract}
In this paper, the robust stabilization for a class of uncertain nonlinear systems is investigated. Based on the Lyapunov-like approach with differential inequalities, a simple linear static control is offered to realize the global exponential stability of such uncertain nonlinear systems. Meanwhile, the guaranteed exponential convergence rate can be correctly estimated. Finally, some numerical simulations are given to demonstrate the feasibility and effectiveness of the obtained results.
\end{abstract}

Key Words: Global exponential stabilization, uncertain nonlinear systems, exponential convergence rate, linear static control

\section{INTRODUCTION}

Control design with implementation of uncertain nonlinear dynamical systems is one of the most challenging areas in systems and control theory. It is well known that uncertainties and nonlinearities often appear in various physical systems and all physical systems are essentially nonlinear in nature. Nonlinear control has been active for many years, and many results concerning with nonlinear control have been proposed. However, it is still difficult to implement nonlinear controllers for practical systems.

Recently, there have several well-developed techniques and methodologies for analyzing uncertain nonlinear systems, such as back stepping approach, fuzzy adaptive control approach, feedback linearization, H-infinity control approach, sliding mode control methodology, LMI approach, singular perturbation method, Lyapunov approach, Riccati equation approach, center manifold theorem, adaptive sliding mode control, adaptive fuzzy-neural-network, and others; see, for example, [1-8] and the references therein.
In this paper, the stabilizability for a class of uncertain nonlinear systems will been considered. Based on the Lyapunoe-like approach with differential inequality, a linear static control will be established to realize the global exponential stability of such uncertain systems. Moreover, the guaranteed exponential convergence rate can be correctly calculated. Several numerical simulations will also be provided to illustrate the use of the main results.

The layout of the rest of this paper is organized as follows. The problem formulation, main result, and controller design are presented in Section 2. In Section 3, numerical simulations with circuit realization are given to illustrate the effectiveness of the developed results. Finally, some conclusions are drawn in Section 4. In what follows, $\Re^{n}$ denotes the n-dimensional real space, $\|x\|$ denotes the Euclidean norm of the vector $x \in \Re^{n},|a|$ denotes the absolute value of a real number a, and $A^{T}$ denotes the transport of the matrix $A$.

\section{PROBLEM FORMULATION AND MAIN RESULTS}

In this paper, we explore the following uncertain nonlinear systems:

$$
\begin{aligned}
& \dot{x}_{1}=\Delta a_{1} x_{1}+\Delta a_{2} x_{2}+\Delta a_{3} x_{4}+\Delta a_{4} x_{3} x_{4} \text {, } \\
& \dot{x}_{2}=\Delta a_{5} x_{1}+\Delta a_{6} x_{2}+\Delta a_{7} x_{3}+\Delta a_{8} x_{4} \\
& +\Delta a_{9} x_{1} x_{3}+\Delta a_{10} u_{1} \\
& \dot{x}_{3}=\Delta a_{11} x_{2}+\Delta a_{12} x_{3}-\Delta a_{9} x_{1} x_{2} \text {, } \\
& \dot{x}_{4}=\Delta a_{13} x_{1}+\Delta a_{14} x_{2}-\Delta a_{4} x_{1} x_{3}+\Delta a_{15} u_{2} \text {, } \\
& {\left[\begin{array}{llll}
x_{1}(0) & x_{2}(0) & x_{3}(0) & x_{4}(0)
\end{array}\right]^{T}} \\
& =\left[\begin{array}{llll}
x_{10} & x_{20} & x_{30} & x_{40}
\end{array}\right]^{T},
\end{aligned}
$$

where $x(t):=\left[\begin{array}{llll}x_{1}(t) & x_{2}(t) & x_{3}(t) & x_{4}(t)\end{array}\right]^{T} \in \mathfrak{R}^{4}$ is the state vector, $u(t):=\left[u_{1}(t) \quad u_{2}(t)\right]^{T} \in \mathfrak{R}^{2}$ is the system control, 
$\left[\begin{array}{llll}x_{10} & x_{20} & x_{30} & x_{40}\end{array}\right]^{T}$ is the initial value, and $\Delta a_{i}, \forall i \in\{1,2, \cdots, 15\}$ indicate uncertain parameters of the system. The hyper-chaotic Pan system is a special case of systems (1) with $u(t)=0, \quad \Delta a_{2}=-\Delta a_{1}=10$, $\Delta a_{5}=28, \Delta a_{8}=-\Delta a_{9}=1, \Delta a_{12}=\frac{-8}{3}, \Delta a_{14}=-10$, and $\Delta a_{i}=0, \quad \forall i \in\{3,4,6,7,10,11,13,15\}$.

The global exponential stabilization and the exponential convergence rate of the system (1) are

\section{Definition 1.}

The uncertain systems (1) are said to be globally exponentially stable if there exist a control $u$ and positive number $\alpha$ satisfying

$$
\|x(t)\| \leq\|x(0)\| \cdot e^{-\alpha t}, \quad \forall t \geq 0 .
$$

In this case, the positive number $\alpha$ is called the exponential convergence rate.

The aim of this paper is to find a simple linear static control such that the global exponential stabilization of uncertain systems (1) can be guaranteed. Meanwhile, an estimate of the exponential explored.

Throughout this paper, we make the following assumption:

(A1) There exist constants $\bar{a}_{i}$ and $a_{i}$ such that $\underline{a_{i}} \leq \Delta a_{i} \leq \bar{a}_{i}, \forall i \in\{1,2, \cdots, 15\}, \quad$ with $b_{i}:=\max \left\{\bar{a}_{i}|,| a_{i}\right\}$, $\underline{a_{i}}>0, \forall i \in\{10,15\}$ and $\bar{a}_{i}<0, \forall i \in\{1,12\}$. defined as follows. convergence rate of such stable systems is also

with $\delta_{1}>0$ and $\delta_{2}>0$. In this case, the guaranteed exponential convergence rate is given by

$\alpha:=\min \left\{\frac{-\bar{a}_{1}}{3}, \quad \delta_{1}, \frac{-\bar{a}_{12}}{2}, \delta_{2}\right\}$.

Proof. Let

$V(x(t)):=x_{1}^{2}(t)+x_{2}^{2}(t)+x_{3}^{2}(t)+x_{4}^{2}(t)$.

The time derivative of $V(x(t))$ along the trajectories of the closed-loop systems (1) with (2) and (A1), is given by

$\dot{V}(x(t))$

$$
\begin{aligned}
= & 2 x_{1} \dot{x}_{1}+2 x_{2} \dot{x}_{2}+2 x_{3} \dot{x}_{3}+2 x_{4} \dot{x}_{4} \\
= & 2 x_{1}\left(\Delta a_{1} x_{1}+\Delta a_{2} x_{2}+\Delta a_{3} x_{4}+\Delta a_{4} x_{3} x_{4}\right) \\
& +2 x_{2}\left(\Delta a_{5} x_{1}+\Delta a_{6} x_{2}+\Delta a_{7} x_{3}+\Delta a_{8} x_{4}\right. \\
& \left.+\Delta a_{9} x_{1} x_{3}-\Delta a_{10} k_{1} x_{2}\right) \\
& +2 x_{3}\left(\Delta a_{11} x_{2}+\Delta a_{12} x_{3}-\Delta a_{9} x_{1} x_{2}\right)
\end{aligned}
$$

$+2 x_{4}\left(\Delta a_{13} x_{1}+\Delta a_{14} x_{2}-\Delta a_{4} x_{1} x_{3}-\Delta a_{15} k_{2} x_{4}\right)$

$\leq 2 \bar{a}_{1} x_{1}^{2}+2 b_{2}\left|x_{1}\right|\left|x_{2}\right|+2 b_{3}\left|x_{1}\right|\left|x_{4}\right|+2 \Delta a_{4} x_{1} x_{3} x_{4}$

$$
+2 b_{5}\left|x_{1}\right|\left|x_{2}\right|+2 b_{6} x_{2}^{2}+2 b_{7}\left|x_{2}\right|\left|x_{3}\right|+2 b_{8}\left|x_{2}\right|\left|x_{4}\right|
$$

$+2 \Delta a_{9} x_{1} x_{2} x_{3}-2 a_{10} k_{1} x_{2}^{2}$

$+2 b_{11}\left|x_{2}\right|\left|x_{3}\right|+2 \bar{a}_{12} x_{3}^{2}-2 \Delta a_{9} x_{1} x_{2} x_{3}$

$+2 b_{13}\left|x_{1}\right|\left|x_{4}\right|+2 b_{14}\left|x_{2}\right|\left|x_{4}\right|-2 \Delta a_{4} x_{1} x_{3} x_{4}$

$-2 a_{15} k_{2} x_{4}^{2}$

$\leq 2\left(\frac{\bar{a}_{1}}{3}+\frac{\bar{a}_{1}}{3}+\frac{\bar{a}_{1}}{3}\right) x_{1}^{2}+2\left(b_{2}+b_{5}\right)\left|x_{1}\right|\left|x_{2}\right|$

$+2\left(b_{3}+b_{13}\right)\left|x_{1}\right|\left|x_{4}\right|+2 b_{6} x_{2}^{2}+2\left(b_{7}+b_{11}\right)\left|x_{2}\right|\left|x_{3}\right|$

$+2\left(b_{8}+b_{14}\right)\left|x_{2}\right|\left|x_{4}\right|+2\left(\frac{\bar{a}_{12}}{2}+\frac{\bar{a}_{12}}{2}\right) x_{3}^{2}$

$-2\left[\frac{-3\left(b_{2}+b_{5}\right)^{2}}{4 \bar{a}_{1}}+\frac{-\left(b_{7}+b_{11}\right)^{2}}{2 \bar{a}_{12}}+b_{6}+\delta_{1}+1\right] x_{2}^{2}$

Now we present the main result for the globally exponential stabilization of uncertain systems (1) via Lyapunov-like theorem with the differential and integral inequalities.

\section{Theorem 1}

The uncertain systems (1) with (A1) realize the globally exponential stabilization under the linear static control

$u=\left[\begin{array}{ll}u_{1} & u_{2}\end{array}\right]^{T}=\left[\begin{array}{ll}-k_{1} x_{2} & -k_{2} x_{4}\end{array}\right]^{T}$,

where

$k_{1} \geq \frac{1}{\underline{a_{10}}} \times$

$\left[\frac{-3\left(b_{2}+b_{5}\right)^{2}}{4 \bar{a}_{1}}+\frac{-\left(b_{7}+b_{11}\right)^{2}}{2 \bar{a}_{12}}+b_{6}+\delta_{1}+1\right]$,

$k_{2} \geq \frac{1}{\underline{a_{15}}}\left[\frac{-3\left(b_{3}+b_{13}\right)^{2}}{4 \bar{a}_{1}}+\frac{\left(b_{8}+b_{14}\right)^{2}}{4}+\delta_{2}\right]$, $-2\left[\frac{-3\left(b_{3}+b_{13}\right)^{2}}{4 \bar{a}_{1}}+\frac{\left(b_{8}+b_{14}\right)^{2}}{4}+\delta_{2}\right] x_{4}^{2}$

$=-2\left[\frac{-\bar{a}_{1}}{3} x_{1}^{2}-\left(b_{2}+b_{5}\right)\left|x_{1}\right|\left|x_{2}\right|+\frac{-3\left(b_{2}+b_{5}\right)^{2}}{4 \bar{a}_{1}} x_{2}^{2}\right]$

$-2\left[\frac{-\bar{a}_{1}}{3} x_{1}^{2}-\left(b_{3}+b_{13}\right)\left|x_{1}\right|\left|x_{4}\right|+\frac{-3\left(b_{3}+b_{13}\right)^{2}}{4 \bar{a}_{1}} x_{4}^{2}\right]$

$-2\left[\frac{-\bar{a}_{12}}{2} x_{3}^{2}-\left(b_{7}+b_{11}\right)\left|x_{2}\right|\left|x_{3}\right|+\frac{-\left(b_{7}+b_{11}\right)^{2}}{2 \bar{a}_{12}} x_{2}^{2}\right]$

$-2\left[x_{2}^{2}-\left(b_{8}+b_{14}\right)\left|x_{2} \| x_{4}\right|+\frac{\left(b_{8}+b_{14}\right)^{2}}{4} x_{2}^{2}\right]$

$-2\left(\frac{-\bar{a}_{1}}{3}\right) x_{1}^{2}-2\left(\frac{-\bar{a}_{12}}{2}\right) x_{3}^{2}-2 \delta_{1} x_{2}^{2}-2 \delta_{2} x_{4}^{2}$

$\leq-2\left(\frac{-\bar{a}_{1}}{3}\right) x_{1}^{2}-2\left(\frac{-\bar{a}_{12}}{2}\right) x_{3}^{2}-2 \delta_{1} x_{2}^{2}-2 \delta_{2} x_{4}^{2}$

$\leq-2\left(\alpha x_{1}^{2}+\alpha x_{2}^{2}+\alpha x_{3}^{2}+\alpha x_{4}^{2}\right)$

$=-2 \alpha V, \quad \forall t \geq 0$. 
Hence, one has

$$
\begin{aligned}
e^{2 \alpha t} \cdot \dot{V}+e^{2 \alpha t} \cdot 2 \alpha V & =\frac{d}{d t}\left[e^{2 \alpha t} \cdot V\right] \\
& \leq 0, \quad \forall t \geq 0 .
\end{aligned}
$$

It results

$$
\begin{aligned}
& \int_{0}^{t} \frac{d}{d \tau}\left[e^{2 \alpha \tau} \cdot V(x(t))\right] d \tau \\
= & e^{2 \alpha t} \cdot V(x(t))-V(x(0)) \\
\leq & \int_{0}^{t} 0 d \tau=0, \quad \forall t \geq 0 .
\end{aligned}
$$

From (4) and (5), it follows

$$
\begin{aligned}
\|x(t)\|^{2} & =V(x(t)) \leq e^{-2 \alpha t} V(x(0)) \\
& =e^{-2 \alpha t}\|x(0)\|^{2}, \quad \forall t \geq 0 .
\end{aligned}
$$

As a consequence, we conclude that $\|x(t)\| \leq e^{-\alpha t}\|x(0)\|, \quad \forall t \geq 0$.

This completes the proof.

\section{NUMERICAL SIMULATIONS}

Consider the uncertain systems (1) with

$$
\begin{aligned}
& -11 \leq \Delta a_{1} \leq-10, \quad 9 \leq \Delta a_{2} \leq 10, \\
& 27 \leq \Delta a_{5} \leq 28, \quad 0 \leq \Delta a_{8} \leq 1, \\
& -1 \leq \Delta a_{9} \leq 1, \quad-3 \leq \Delta a_{12} \leq \frac{-8}{3}, \\
& -10 \leq \Delta a_{14} \leq-9, \quad 1 \leq \Delta a_{10}, \Delta a_{15} \leq 2, \\
& \Delta a_{i}=0, \quad \forall i \in\{3,4,6,7,11,13\} .
\end{aligned}
$$

By comparing (A1) and (6) with selecting the parameters of

$\left(\bar{a}_{1}, \underline{a_{10}}, \bar{a}_{12}, \underline{a_{15}}\right)=\left(-10,1, \frac{-8}{3}, 1\right)$,

$\left(b_{2}, b_{5}, b_{7}, b_{11}, b_{6}\right)=(10,28,0,0,0)$,

$\left(b_{3}, b_{13}, b_{8}, b_{14}\right)=(0,0,1,10)$,

(A1) is evidently satisfied. With the choice $\delta_{1}=\delta_{2}=1$ in (2), it can be obtained that $u=\left[\begin{array}{ll}u_{1} & u_{2}\end{array}\right]^{T}=\left[\begin{array}{ll}-111 x_{2} & -32 x_{4}\end{array}\right]^{T}$.

As a consequence, by Theorem 1, we conclude that the uncertain systems (1) with (6) are globally exponentially stable under the linear static control of (7). Besides, from (3), the guaranteed exponential convergence rate is given by $\alpha=1$. The typical state trajectories of the uncontrolled system and the feedback-controlled system are depicted in Figure 1 and Figure 2, respectively. In addition, the control signals and the electronic circuit to realize such a control law are depicted in Figure 3 and Figure 4, respectively.

\section{CONCLUSION}

In this paper, the robust stabilization for a class of uncertain nonlinear systems has been explored. Based on the Lyapunov-like approach with differential inequalities, a simple linear static control has been presented to realize the global exponential stability of such uncertain nonlinear systems. Meanwhile, the guaranteed exponential convergence rate can be correctly calculated. Finally, some numerical simulations have been offered to demonstrate the feasibility and effectiveness of the obtained results.

\section{ACKNOWLEDGEMENT}

The author thanks the Ministry of Science and Technology of Republic of China for supporting this work under grants MOST 106-2221-E-214-007, MOST 106-2813-C-214-025-E, and MOST 107-2221E-214-030. Furthermore, the author is grateful to Chair Professor Jer-Guang Hsieh for the useful comments.

\section{REFERENCES}

1. A. Bayas, I. Škrjanc, and D. Sáez, "Design of fuzzy robust control strategies for a distributed solar collector field," Applied Soft Computing, vol. 71, pp. 1009-1019, 2018.

2. Z.M. Li and X.H. Chang, "Robust $\mathrm{H} \infty$ control for networked control systems with randomly occurring uncertainties: Observer-based case," ISA Transactions, vol. 83, pp. 13-24, 2018.

3. R.X. Zhong, C. Chen, Y.P. Huang, A. Sumalee, and D.B. $\mathrm{Xu}$, "Robust perimeter control for two urban regions with macroscopic fundamental diagrams: A control-Lyapunov function approach," Transportation Research Part B: Methodological, vol. 117, pp. 687-707, 2018.

4. F. Yue and X. Li, "Robust adaptive integral backstepping control for opto-electronic tracking system based on modified LuGre friction model," ISA Transactions, vol. 80, pp. 312-321, 2018.

5. N. Adhikary and C. Mahanta, "Sliding mode control of position commanded robot manipulators," Control Engineering Practice, vol. 81, pp. 183-198, 2018.

6. A. Ayadi, M. Smaoui, S. Aloui, S. Hajji, and M. Farza, "Adaptive sliding mode control with moving surface: Experimental validation for electropneumatic system," Mechanical Systems and Signal Processing, vol. 109, pp. 27-44, 2018. 
International Journal of Trend in Scientific Research and Development (IJTSRD) ISSN: 2456-6470

7. Y. Solgi and S. Ganjefar, "Variable structure fuzzy wavelet neural network controller for complex nonlinear systems," Applied Soft Computing, vol. 64, pp. 674-685, 2018.

8. J.F. Qiao, Y. Hou, L. Zhang, and H.G. Han, "Adaptive fuzzy neural network control of wastewater treatment process with multiobjective operation," Neurocomputing, vol. 275, pp. 383393, 2018.

9. A. Erfani, S. Rezaei, M. Pourseifi, and H. Derili, "Optimal control in teleoperation systems with time delay: A singular perturbation approach," Journal of Computational and Applied Mathematics, vol. 338, pp. 168-184, 2018.

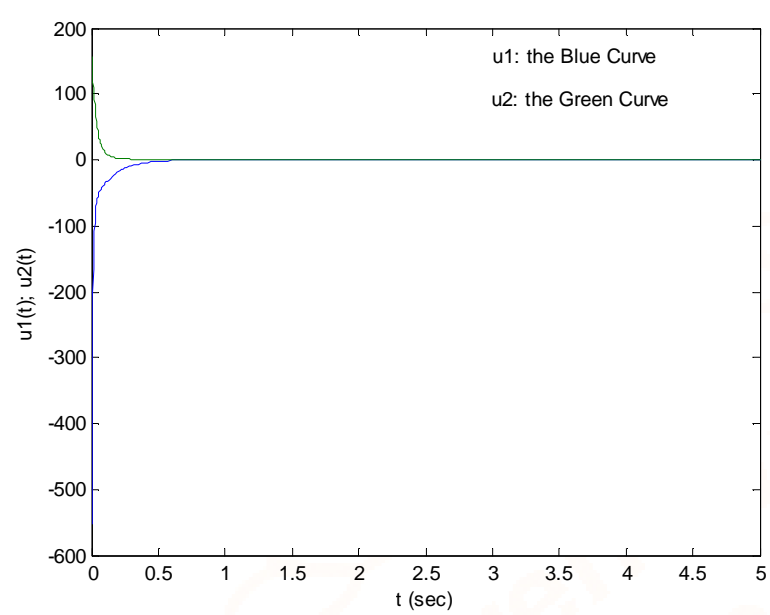

Figure 3: Control signals of $u_{1}(t)$ and $u_{2}(t)$.

10. H. Gritli and S. Belghith, "Robust feedback control of the underactuated Inertia Wheel Inverted Pendulum under parametric uncertainties and subject to external disturbances: LMI formulation," Journal of the Franklin Institute, vol. 355, pp. 9150-9191, 2018.

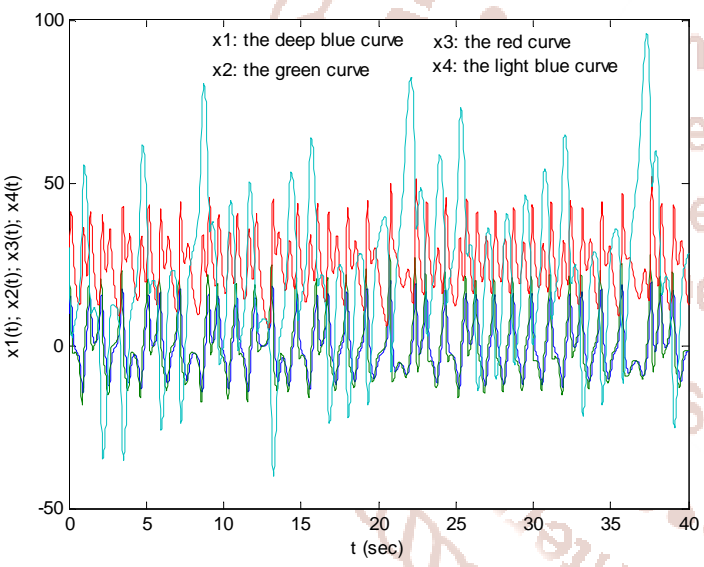

Figure 1: Typical state trajectories of the system (1) with (6) and $u=0$.

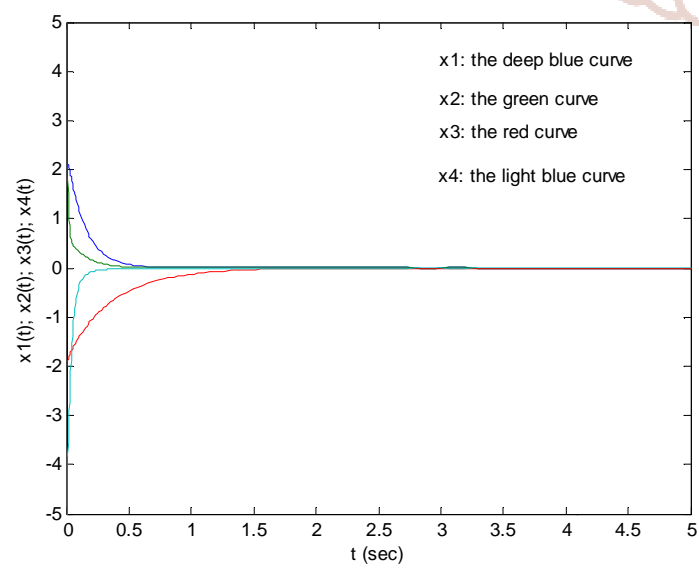

Figure 4: The diagram of implementation of controller, where $R 1=R 3=1 \mathrm{k} \Omega, R 2=111 \mathrm{k} \Omega$, and $R 4=32 k \Omega$.

Figure 2: Typical state trajectories of the feedbackcontrolled system of (1) with (6) and (7). 